\title{
Cummins/ORNL-FEERC CRADA: NOx Control \& Measurement Technology for Heavy-Duty Diesel Engines
}

(Agreement \#: 10030*)

Oak Ridge National Laboratory: W.P. Partridge (PI), J.-S. Choi, J. Parks

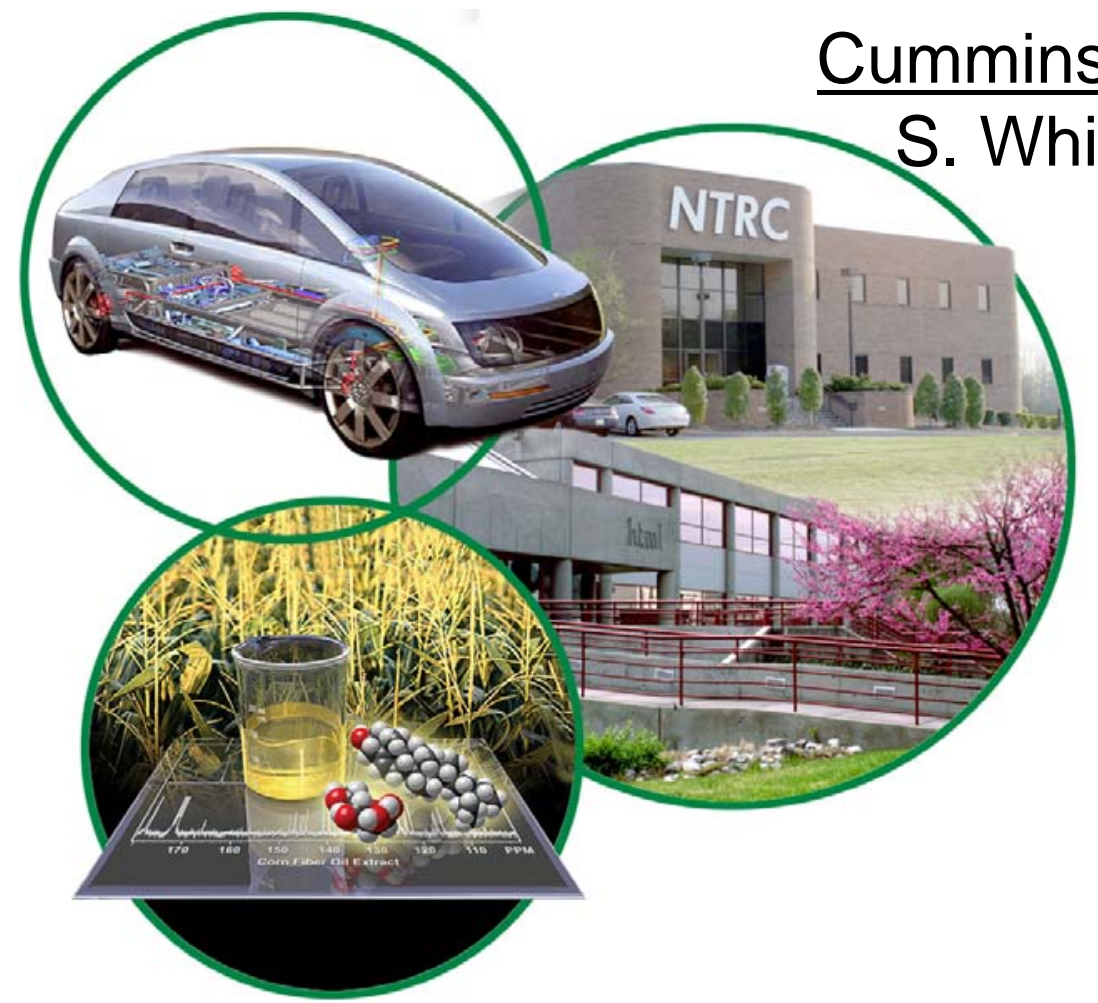

Presenter: Bill Partridge partridgewp@ornl.gov

Vehicle Technologies Program Annual Merit Review February 26, 2008, Bethesda, MD

U.S. DOE Program Management Team: Ken Howden, Gurpreet Singh, Steve Goguen

*This presentation does not contain any proprietary or confidential information.

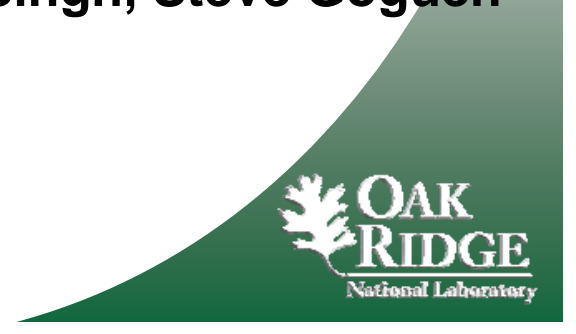


Assist Cummins in addressing barriers

to transportation-market penetration

of fuel-efficient diesel engines

\& by doing so

generate useful knowledge

and enable products

with broad public benefit

Major Focus for FY2008:

- Oil Dilution (from engine-managed catalyst regeneration)

- LNT-Catalyst Ammonia Chemistry 
- Feedback was very positive

- Highest score achieved in three areas

- Relevance

- Technical Accomplishments

- Tech Transfer

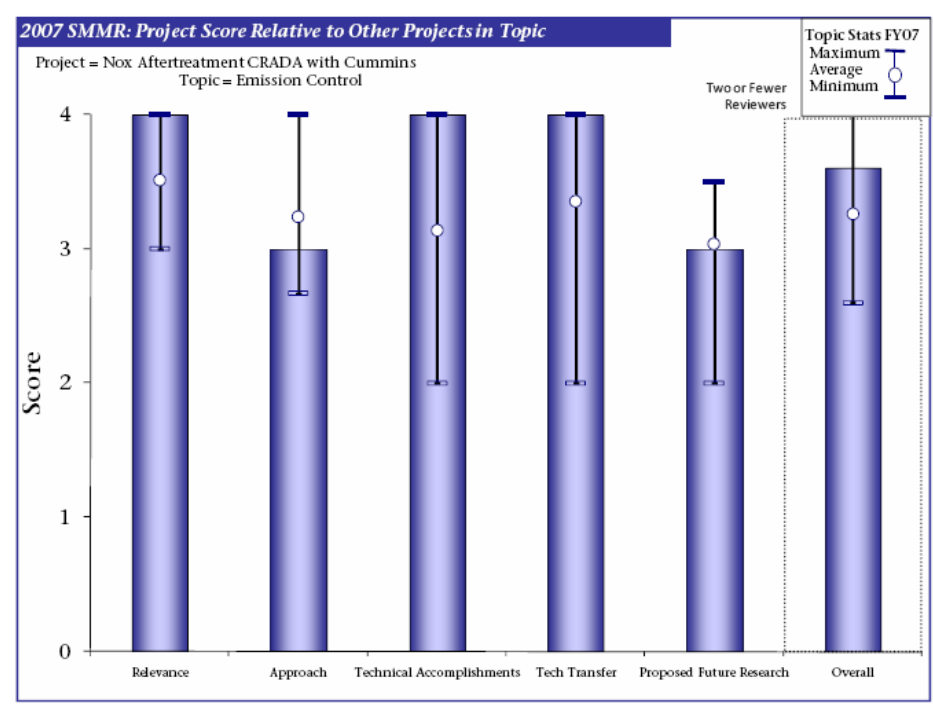

- "A useful industry partnership that has shown good progress and results."

- No weaknesses or recommendations were cited.

- Previous review recommendations:

- Using fully formulated catalyst (implemented in '07)

- Quantifying $\mathrm{H}_{2} \mathrm{~S}, \mathrm{NH}_{3}, \mathrm{~N}_{2} \mathrm{O}$ and $\mathrm{SO}_{2}$ distributions (implemented in '07 except $\mathrm{NH}_{3}$ )

- Major effort in FY2008 to quantify $\mathrm{NH}_{3}$ chemistry 


\section{CRADA Addresses Multiple DOEIVT Barriers}

\section{Oil Dilution (Engine System Section)}

- Can result from emissions control system management and operation

- Oil dilution can cause durability issues

- Conventional methodologies slow development

\section{LNT Ammonia Chemistry (Instrumentation \& Bench Section)}

- $\mathrm{NH}_{3}$ must be controlled in viable catalyst systems:

- Avoid $\mathrm{NH}_{3}$ slip

- Manage generation and utilization in LNT and hybrid LNT-SCR systems

Specific DOEIVT Multi-Year Program Plan Barriers Addressed :

- 'Emissions. The key barriers ... incomplete development of aftertreatment technology, especially for NOx;.."

- 'Durability. .. system has to perform effectively for 120,000 miles...' 


\section{Oil Dilution Research}




\section{Performance Measures - Oil Dilution}

Conventional methodologies:

- bottleneck development

- require extractive sampling

- off-line (often off-site) analysis

Performance measures designed to streamline development:

- Fast measurement $\sim 15$ min

- On-engine measurements

- Real-time feedback 


\section{Approach - Oil Dilution}

- Inexpensive \& compact laser-pointer sources

- Fluorescence of commercial diesel-fuel dye

- Two fiber-based designs implemented

- Sample-point agile optical fiber design

- Engine-cell safe closed system design

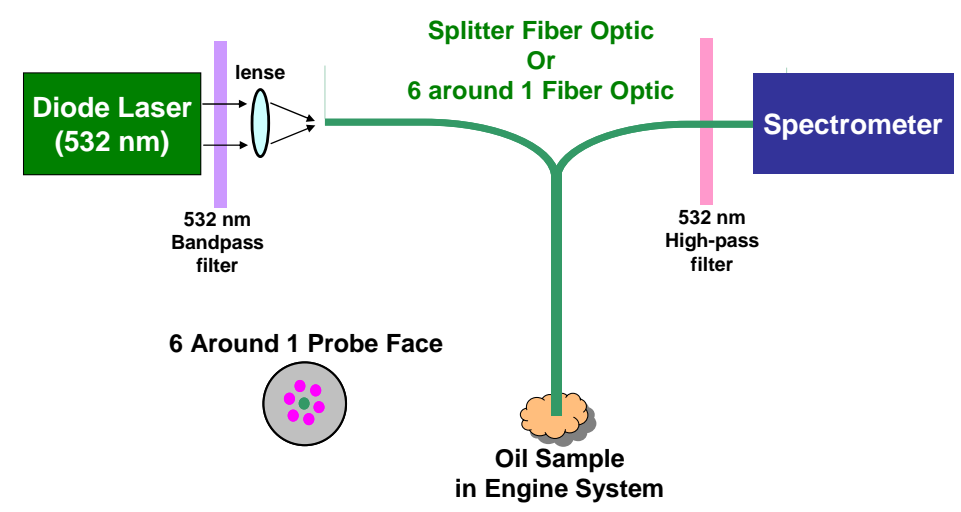

- Demonstrate on ORNL research diesel

- Flexible engine control system

- Extract samples for ASTM method comparison
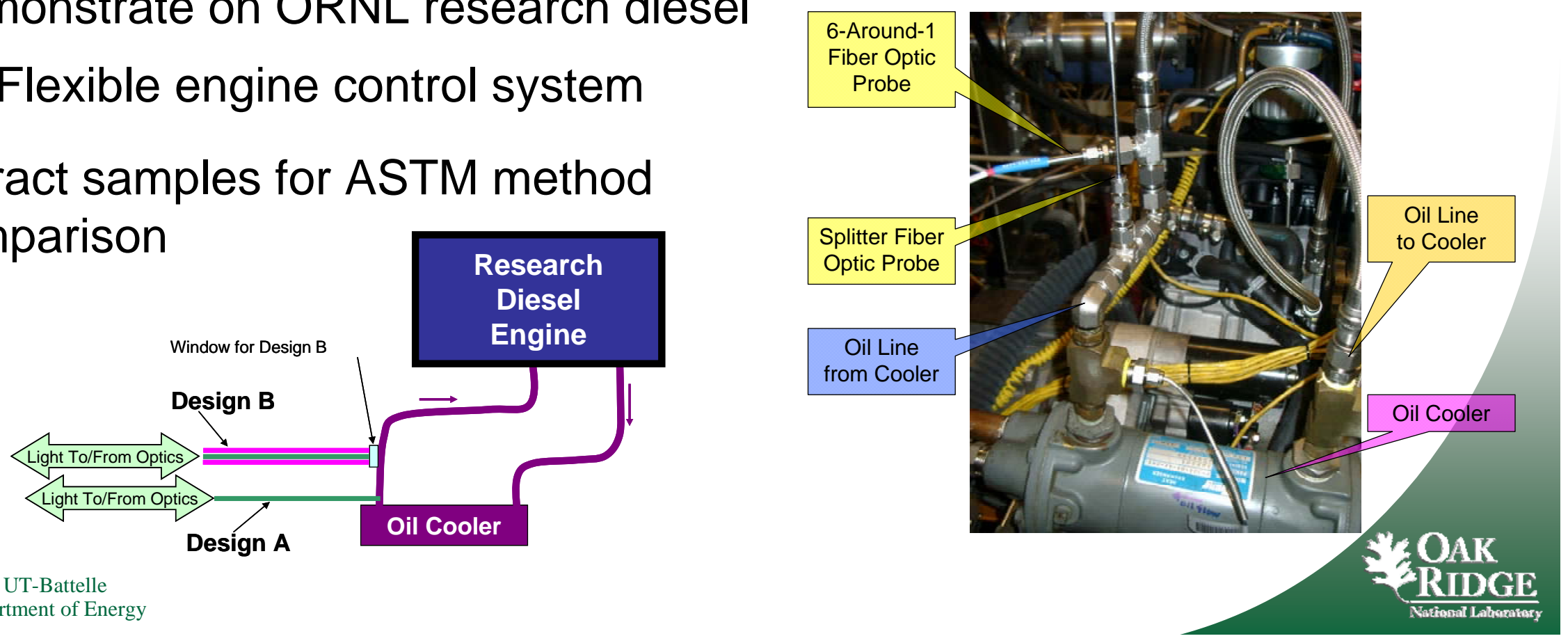


\section{Results - Oil Dilution}
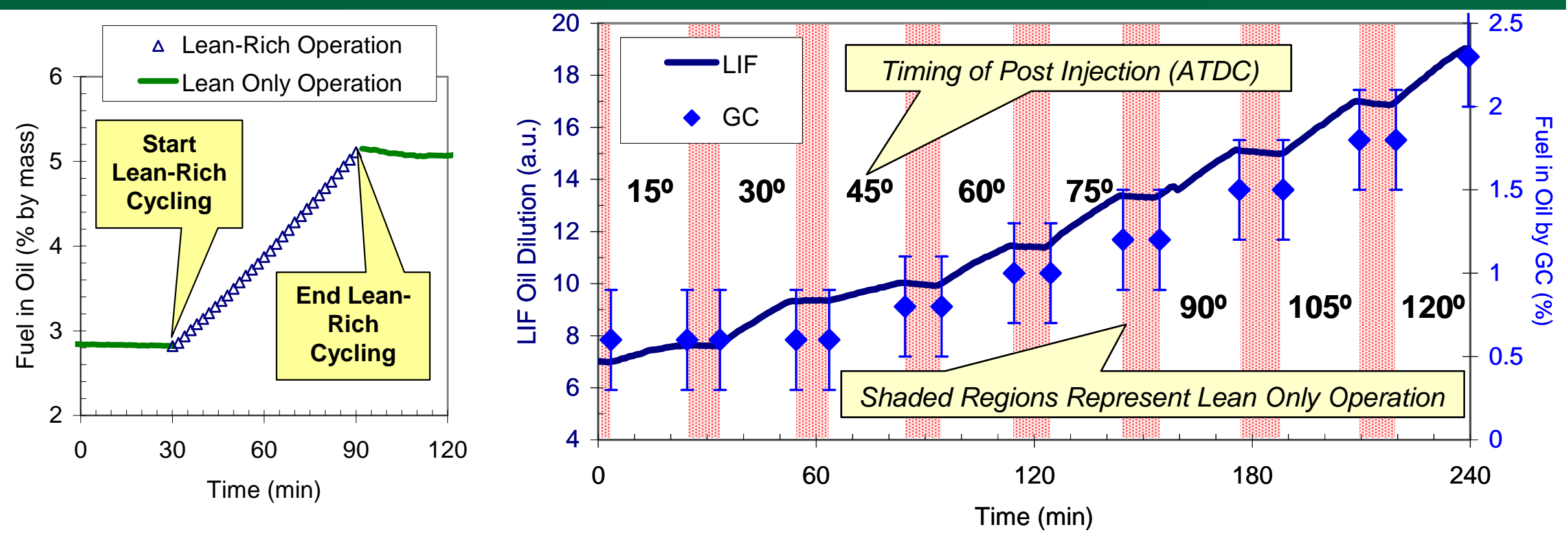

- Slope indicates relative oil dilution rate

- Real-time on-engine feedback of oil dilution

- <15 min feedback time

- Laser-Induced Fluorescence (LIF) method

- trends with ASTM

- more sensitive than ASTM

- LIF Oil Dilution diagnostic realizes performance measures 


\section{LNT Ammonia Chemistry Research}




\section{Performance Measures - LNT Ammonia Chemistry}

Ammonia is relevant to diesel product development:

- $\mathrm{NH}_{3}$ slip management

- System design \& calibration

- On-board detection of system state

- Cummins development emphasizes fundamental knowledge

Ammonia is difficult to measure w/ SpaciMS:

- Interferences with $\mathrm{N}_{2}, \mathrm{H}_{2} \mathrm{O}, \mathrm{NOx}$

- $\mathrm{NH}_{3}$ is sticky

- Variable and different elution times

Performance Measures address diagnostic and information needs:

- Enable SpaciMS measurement of $\mathrm{NH}_{3}$ inside operating LNT catalysts

- Map transient $\mathrm{NH}_{3}$ distribution through catalyst

- Clarify $\mathrm{NH}_{3}$ formation and utilization in LNT chemistry 


\section{Approach - LNT Ammonia Chemistry}

- Catalyst core (3/4" x 3") on bench reactor

- Washcoat: $\mathrm{Pt} / \mathrm{Ba} / \mathrm{Al}_{2} \mathrm{O}_{3}$ model catalyst

- No cerium - oxygen-storage component

- CLEERS standard short cycling:

- 60-s lean: 300ppm NO + 10\% $\mathrm{O}_{2}$

- 5-s rich: $2 \% \mathrm{H}_{2}$

- Common: $5 \% \mathrm{H}_{2} \mathrm{O}+5 \% \mathrm{CO}_{2}+$ Ar balance

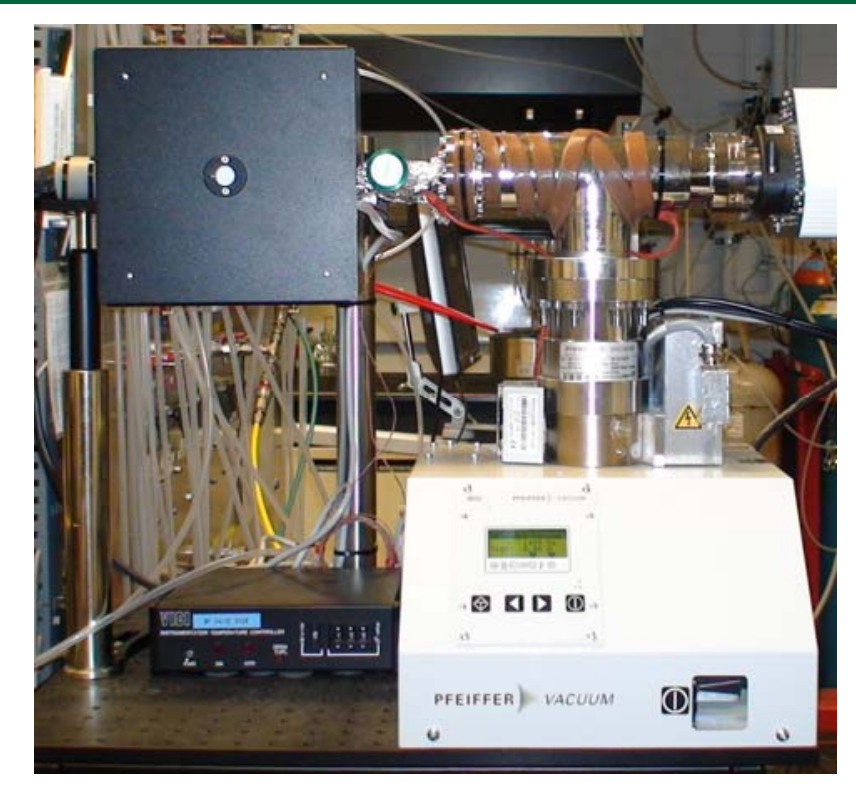

- Resolve species distributions along catalyst channel

- SpaciMS

- $\mathrm{NH}_{3}$ generation and utilization

- NOx, $\mathrm{N}_{2}$ and $\mathrm{H}_{2}$

- Analyze distributed performance

- Phase/timing of species puffs

- Selectivity
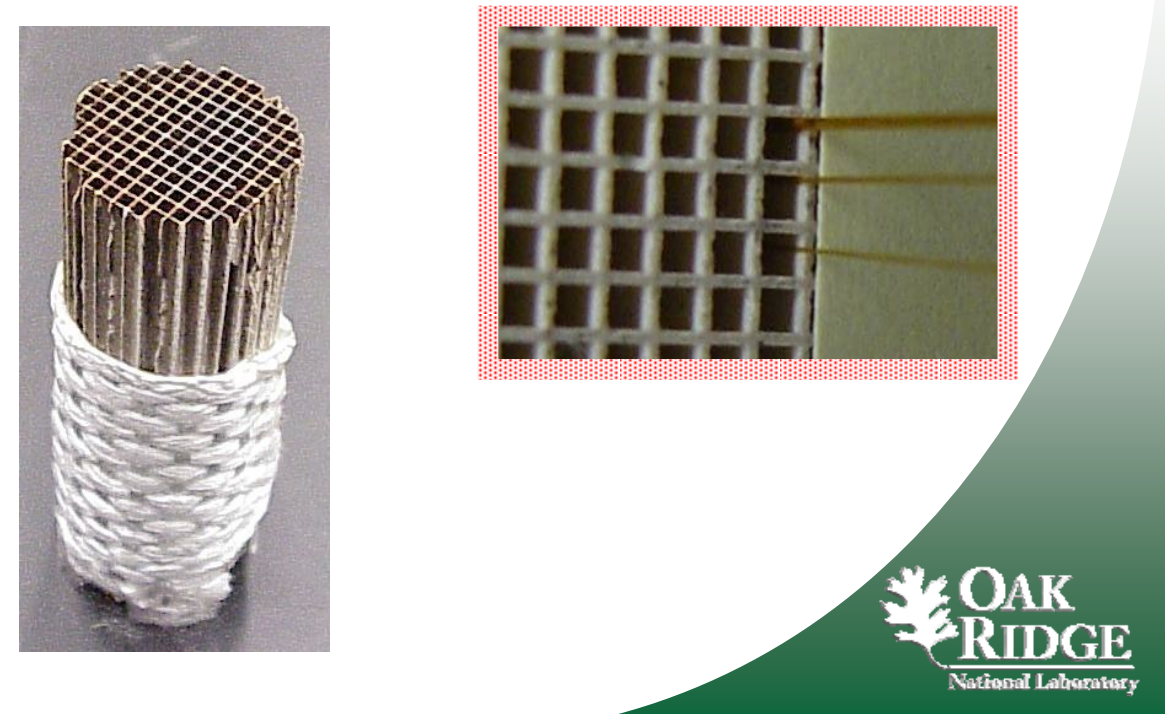


\section{Results - LNT Ammonia Chemistry}
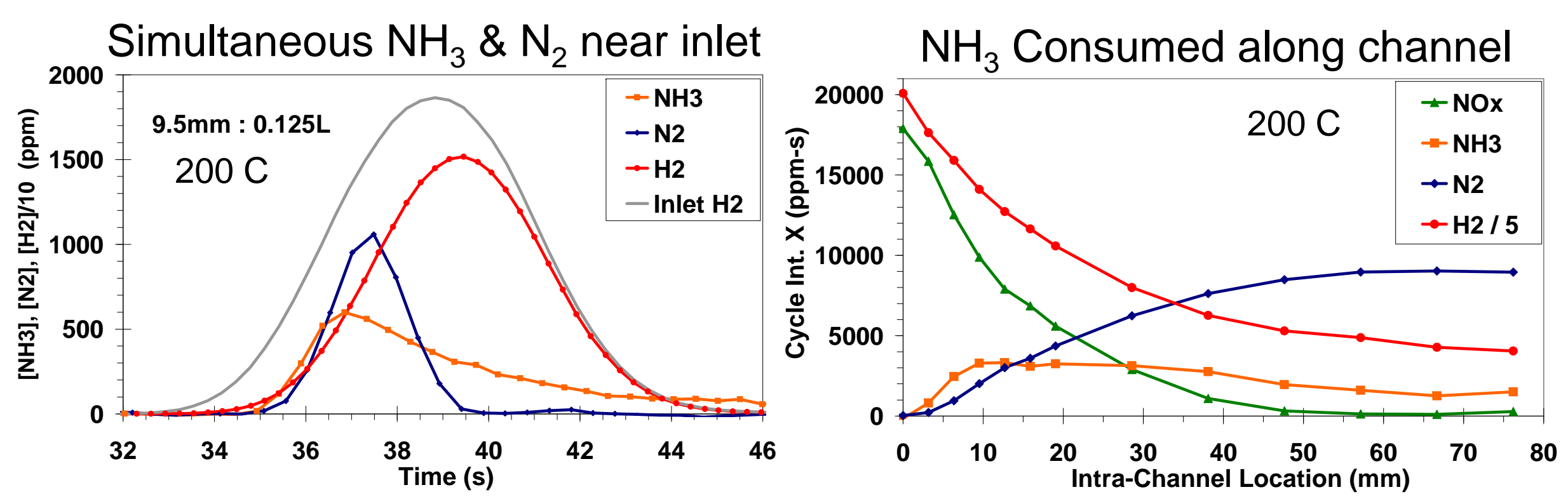

- Simultaneous $\mathrm{N}_{2}$ \& $\mathrm{NH}_{3}$ generation at catalyst front

- $\mathrm{NH}_{3}$ shifts to later times along catalyst length

- $\mathrm{NH}_{3}$ consumed along catalyst length along with $\mathrm{H}_{2}$

SpaciMS $\mathrm{NH}_{3}$ measurements demonstrated Must account for $\mathrm{NH}_{3}$ regeneration role 


\section{Results - Vis-à-vis Literature Ammonia Chemistry}
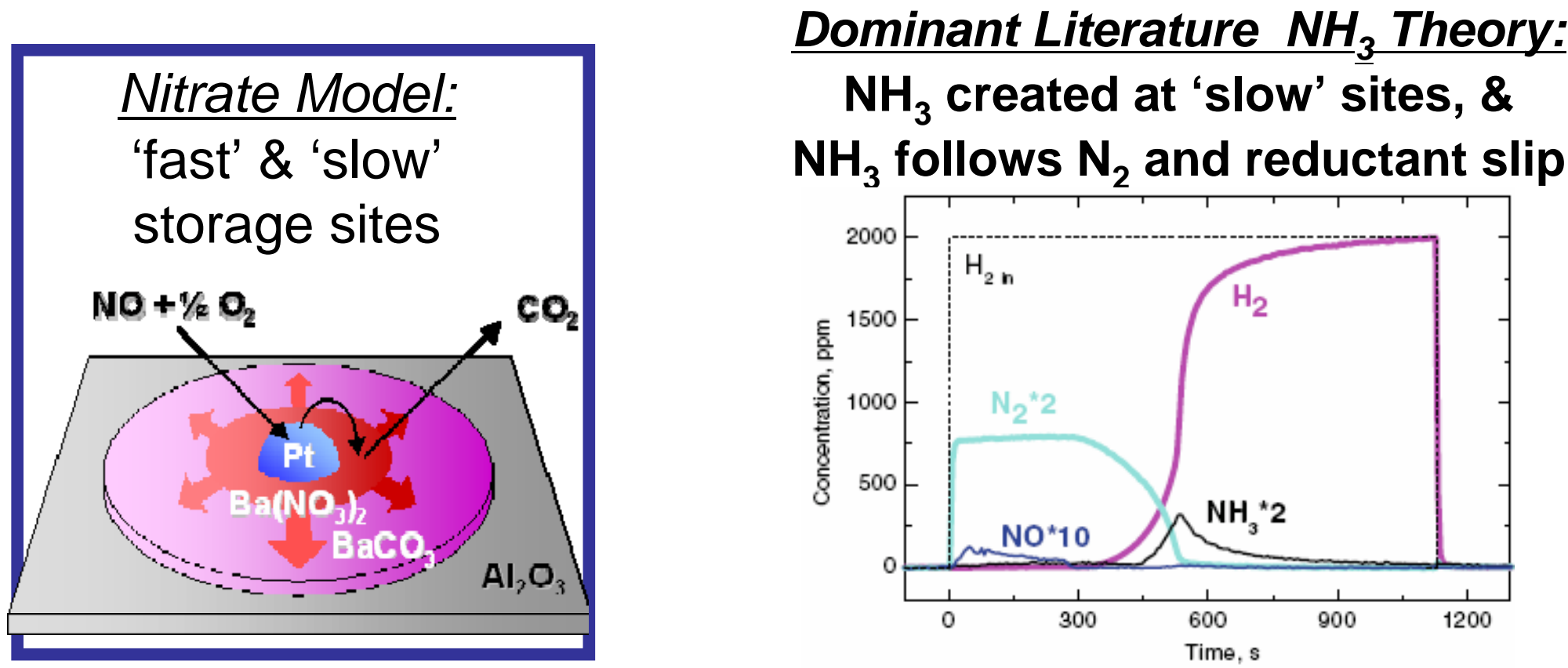

Nova et al. (2007) Topics in Catalysis

- Our measurements show same sequence at outlet

- But simultaneous $\mathrm{NH}_{3}$ and $\mathrm{N}_{2}$ inside catalyst

$\mathrm{NH}_{3}$ doesn't always follow $\mathrm{N}_{2}$

Literature $\mathrm{NH}_{3}$ model incomplete

$\mathrm{NH}_{3}$ not always from 'slow' sites 


\section{Technology Transfer}

Via CRADA:

- All CRADA activities impact Cummins' product development

- CRADA instrumental in the commercialization of the 2007 Dodge Ram engine-catalyst system

Outside of CRADA:

- Coordination with CLEERS impacts broad range of DOE interests

- Delphi improved reformer-catalyst models based on SpaciMS analysis

- Multiple industry inquiries into Oil Dilution diagnostic

- Hiden Analytical marketing commercial SpaciMS

- 2007 Cummins patent expands SpaciMS capabilities
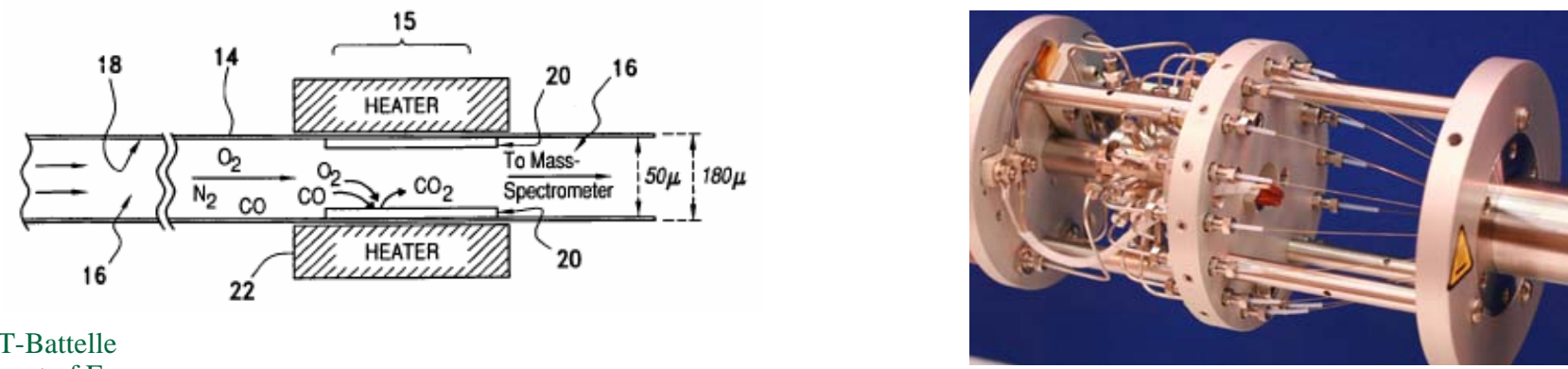


\section{Publications, Presentations, Patents}

\section{Publications:}

- J.-S. Choi, W.P. Partridge, J.A. Pihl, and C.S. Daw, "Sulfur and temperature effects on the spatial distribution of reactions inside a lean $\mathrm{NO}_{x}$ trap and resulting changes in global performance", Catalysis Today, doi:10.1016/j.cattod.2008.01.008 (2008).

- J.-S. Choi, W.P. Partridge, and C.S. Daw, "Sulfur impact on NO storage, oxygen storage and ammonia breakthrough during cyclic lean/rich operation of a commercial lean $\mathrm{NO}_{\mathrm{x}}$ trap", Applied Catalysis B: Environmental 77, 145-156 (2007).

- Jim Parks, Bill Partridge and Shawn Whitacre "Rapid In Situ Measurement of Fuel Dilution of Oil in a Diesel Engine using Laser-Induced Fluorescence Spectroscopy," Society of Automotive Engineers paper 2007-01-4108, 2007.

\section{Presentations:}

- J.-S. Choi, W.P. Partridge, J.A. Pihl, and C.S. Daw, "Sulfur effects on spatiotemporal distribution of reactions in a commercial lean NO trap", AIChE National Meeting, Salt Lake City, UT, November 4-9, 2007.

- Jim Parks, Bill Partridge, and Shawn Whitacre, Rapid In Situ Measurement of Fuel Dilution of Oil in a Diesel Engine Using Laser-Induced Fluorescence Spectroscopy, presented at the SAE Powertrain and Fluid Systems Conference in Chicago, IL on October 29-31, 2007.

- J.-S. Choi, W.P. Partridge, and C.S. Daw, "Assessing a commercial lean NO trap performance via spatiotemporal species profile measurements", North American Meeting (NAM) of the North American Catalysis Society, Houston, TX, June 17-22, 2007.

- W.P. Partridge, J.-S. Choi, C.S. Daw "Distributed Impact of Sulfation on LNT Catalyst Reactions," 10th DOE Crosscut Workshop on Lean Emissions Reduction Simulation, University of Michigan, Dearborn, Michigan, May 2nd, 2007.

\section{Patent:}

- $\quad$ N.W. Currier, A. Yezerets, US Patent Number US 7,211,793 B2, Mass Spectrometry System and Method, May 1, 2007.

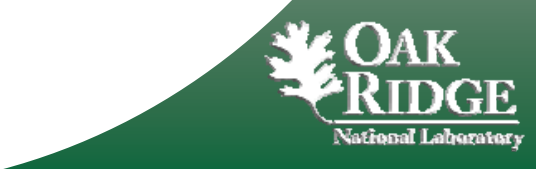




\section{Future Activities}

\section{Engine-Systems Section:}

- Apply oil-dilution diagnostic on development $V$ engine at Cummins (scheduled - March 2008)

- Develop and apply methods to quantify cylinder and cycle dispersion

\section{Instrumentation \& Bench Section:}

- Characterize $\mathrm{NH}_{3}$ chemistry in LNT with oxygen-storage component (Umicore, CLEERS reference catalyst)

- Characterize sulfation impact on LNT catalyst reactions, including $\mathrm{NH}_{3}$ formation and utilization and water-gas-shift 


\section{Summary}

- Relevance to DOE Objectives

- Reduce fuel consumption by enabling diesel market penetration

- Approach

- Real-time on-engine measurement of oil dilution

- Measure evolution of $\mathrm{NH}_{3}$ generation and utilization inside catalyst

- Technical Accomplishments

- Streamline engine calibration

- Enable SpaciMS $\mathrm{NH}_{3}$ measurements \& clarify LNT catalyst reactions

- Enable improved modeling of LNT and hybrid LNT-SCR systems

- Technology Transfer

- Broad based transfer via Cummins products \& outside CRADA

- Future Plans

- Measure cylinder and cycle dispersion on development engines

- Quantify distributed impact of sulfation on $\mathrm{NH}_{3} \mathrm{LNT}$ reactions

- Designed to address Cummins' technical barriers 